\title{
18 Suicidal behavior in Indian adolescents
}

\author{
Diana Samuel and Leo Sher
}

Suicide is both a public and mental health problem as it is a leading cause of deaths, especially among adolescents. Two factors that contribute to adolescents who commit suicide are having a primary mood disorder and/or substance use. In the Indian culture, the family unit has both a positive and negative impact on suicide. The family serves as a protective factor providing a strong support for the individual, but alternately creating an inseparable individual when seeking mental health care which often complicates the situation. Due to the stigma, Indians typically perceive having a mental illness as shameful. Religion is integral to the Indian culture so much so that individuals often use herbal remedies, seek help from religious leaders and attend religious establishments prior to obtaining a mental health evaluation in those that were subsequently deemed as mentally ill. Despite suicides being underreported and misdiagnosed in India, the highest rates are among those less than 30 years old. The methods most commonly used to commit suicide in India include use of poison (often pesticides), hanging, burning and drowning. When immigrating, Indians tend to switch the methods they use to commit suicide from use of poison to hanging which may reflect a lack of availability of poison or the influence of the host culture. Considering the high suicide rates in adolescents, the importance of providing psychoeducation, restricting access to lethal means, and promoting social integration in immigrants are ways we may be able to help prevent suicides in Indian adolescents.

\subsection{Introduction}

Suicide is the act of intentionally causing one's own death. Suicide attempts as defined by Kar are "any act of self-damage inflicted with self-destructive intentions" (1). The factors that contribute to any particular suicide are diverse and complex. Considering that suicide is the third leading cause of death among those between the ages of 15-24 years (2), it is one that must be explored further. In 2011, in the US alone, $15.8 \%$ of high school students had seriously considered attempting suicide during the 12 months before the survey (3). During the same time, $12.8 \%$ of students had made a plan of how they would commit suicide and $7.8 \%$ had attempted suicide at least once (3). Approximately $50-75 \%$ of children and adolescents who commit suicide have had a mood disorder, most commonly major depression (4). About one quarter to twothirds of adolescents who commit suicide received a diagnosis of substance abuse or dependence $(5,6)$. The stigma associated with mental illness is often a significant barrier to obtaining psychiatric care. When considering the limited mental health resources in countries such as India, it is not surprising that suicide rates for instance may be underreported and/or misclassified in order to prevent further shame to the 
family. Despite the continuous and growing impact of urbanization, secularization, and Westernization, the traditional joint household, both in ideal and in practice, remains the primary social force in the lives of most Indians.

\subsection{Indian psyche}

Indian psyche is distinct as it has been influenced by various invaders into the country, collectivism and interdependence (7). In India, people learn the essential themes of cultural life within the bosom of a family. In most of the country, the basic units of society are the patrilineal family unit and wider kinship groupings. The most widely desired residential unit is the joint family, ideally consisting of three or four patrilineally related generations, all living under one roof, working, eating, worshiping, and cooperating together in mutually beneficial social and economic activities. Patrilineal joint families include men related through the male lineage, along with their wives and children. Most young women expect to live with their husband's relatives after marriage, while retaining important bonds with their biological families. Indians consider the relationships within a family very intimate, spread over several people including grandparents, parents, uncles, aunts and siblings, thus often making the parents not the sole guardians of the child (7). With age, a series of similar relationships of varying intensities and duration develop and most often, consequently most Indians do not assume full individuality (7). Therefore, unlike the self-reliant, independent nature that is most often nurtured and even idealized in individuals of the Western world, one of the core values of Indians is based on intimacy, family security and its stability (7). The emotional interdependence that family members feel with each other is often so intense that the family becomes almost an organic unit. Ego boundaries are permeable to others in the family, and any notion of a separate self is often dominated by a sense of what psychoanalyst Alan Roland has termed a more inclusive "familial self." Interpersonal empathy, closeness, loyalty, and interdependency are all crucial to life within the family. It is the intimate nature of the family unit that forms a practically inseparable indentify from the individual making it impossible to separate when attempting to treat a person with mental illness (7).

Nonetheless, it is this same family that provides the support that is protective against suicide. Family support has been shown to be a positive factor for those that are at risk for suicide and also integral in the treatment of these individuals. In one study of adolescents that were at risk for suicide in US adolescents with higher levels of family support had significantly higher levels of self-esteem and self-esteem was found to be a protective factor against suicide (8). Self-esteem is formed when one recognizes themselves as competent and worthy which subsequently creates a sense of self-acceptance, self-respect, and satisfaction with one's self and life (8). Feeling connected to the family may reduce feelings of social isolation and loneliness which are often antecedents to suicide behaviors. Family support creates a feeling that 
one is cared for, wanted and loved by its members which diminishes the likelihood that an adolescent will consider suicide as a "rational" alternative for solving their problems (9).

Religion is an integral aspect of life in India, so far as that religious prayers and healers often play a role in the treatment of all illnesses, including mental illness (10). In one study, it was found that ninety percent of the time that those who were studied had experienced any type of symptom of illness, they initially relied entirely on home remedies and other forms of self-treatment (11). This implies that religious beliefs impact the way one perceives illness in India, whether it be physical or mental ailments. In addition to one's religion, educational level, caste and social class, religious affiliation as well as religious attendance affected the psychiatric care sought by those individuals (10). In a study conducted in North India, 84\% of patients eventually seen in a psychiatric institution had been to a local temple prior to obtaining treatment (12) which may be due to having a poor understanding of mental illness or rather a denial of the actual illness. In terms of those people that saw religious healers for mental illness prior to seeking an official psychiatric consultation, the highest rates of psychiatric diagnoses were in the group diagnosed as having schizophrenia and delusional disorders (10). This implies that many individuals with chronic mental illness may not initially recognize their psychiatric illness as being purely psychiatric or they may feel that their illness is better treated through religious means, at least initially.

\subsection{Suicide in India}

The Indian subcontinent encompasses eight distinct countries, India, Pakistan, Afghanistan, Sri Lanka, Bangladesh, Nepal, Bhutan, and the Maldives and collectively, these countries comprise 1.3 billion people. Information gathered on suicide on the Indian subcontinent comes mainly from India, Sri Lanka and Pakistan as the remaining countries have poorly developed mental health services and consequently, mental health issues including suicide is given little importance when compared to other priorities such as infectious diseases and sanitation. Some of the problems in obtaining records for suicides in countries of the Indian subcontinent include legal, social and religious reasons (13). For example, suicide is considered a criminal act in Pakistan and punishable with a jail term and heavy financial penalty. This has led to both suicides and attempted suicides being underdiagnosed and underreported (14). Until 1994, attempting suicide was a punishable act in India (13). Under section 309 of the Indian Penal Code from 1833 it states, "Whoever attempts to commit suicide and does any act towards the commission of such an offence shall be punished with simple imprisonment for a term which may extend to 1 year, or with a fine or with both” (15). It was only in 1994 that the Supreme Court of India found this code was unconstitutional and Justice Rajinder Sachar of the Delhi High Court said in his 
judgment: "Instead of society hanging its head in shame that there should be such social strains that a young man should be driven to commit suicide, it compounds its inadequacy by treating the boy as a criminal. Instead of sending him to the psychiatric clinic it gleefully sends him to mingle with criminals, as if trying its best to see that in future he does fall foul of the punitive section of the Penal Code" (15). In 1996, the Supreme Court held that the right to life in Article 21 of the Constitution did not include the right to die and court struck down Section 309 of the Penal as being a violation of Article 21 and thus unconstitutional (15). Therefore, suicide attempts and the abetting of suicide continue to be misdemeanors in India and a person convicted of an offense of attempted suicide may be indicted to imprisonment for a term not exceeding 1 year, or a fine, or both (15).

From 1978 to 1990, there was an increase of 41.3\% in suicide from 6.4 to 8.9 (per 100,000). This attributes to a growth rate of 4.1\% per year from 1980 to 1990 in suicide. This rate of suicide increase in India during this time is comparable with the global increase in suicide during the same time (16). In 2008, 125,017 people were recorded to have committed suicide, which was a 1.9\% increase from the previous year of 122,637 suicides (17). Even within India there are wide variations in the suicide rates such that the southern states (Kerala, Karnataka, Andhra Pradesh and Tamil Nadu) having a rate greater than 15 per 100,000 while in the northern states (Punjab, Uttar, Pradesh, Bihar and Jammu and Kashmir) the suicide rate is less than 3 per 100,000 (18). This pattern has remained stable within the last 20 years. Some factors that may contribute to this variation are that in the southern states, there is higher literacy, better reporting system, and higher socioeconomic status (18).

The highest percentage of people who commit suicide in India (37.8\%) are below 30 years of age (19). One out of every three of reported suicides every 15 minutes in India were committed by a youth between the ages of 15-29 (19). Kerala, well-known for being India's first fully literate state, has the highest number of suicides with 32 people committing suicide in this state every day (19). Compared to the US where the overall rate of suicide is about 11 deaths per 100,000 persons, it remains slightly higher then the 10.5 suicides reported in India (20, 21). However, this difference may be better attributed to underreporting rather then a true difference according to many studies, but is unclear.

Due to the stigma associated with mental illness, it is thought that the actual suicide rate in India may be higher than that reported due to concern about the harm to family reputation or police involvement (22). In 2010, it was reported that $33.1 \%$ of suicides were accounted for by ingestion of poison, $31.4 \%$ by hanging, $8.8 \%$ by fire/"selfimmolation" and $6.2 \%$ by drowning (23). Also, it is possible and questionable whether the suicides recorded as acts of "self-immolation" may be underestimated and that those deaths reported as accidental deaths due to fire may be due to suicides (22). It has been suggested that the annual suicide rate could be six to nine times the official rate (18) which is likely due to non-reporting, underreporting and misclassification due to stigmas, religious sanctions, legal issues and insufficient registration systems (24). 
Burning has also been a method used as a means of successful suicide among young women (16) with about 30\% of immigrant Indians committing suicide by burning (25). A suicidology study, conducted from March to October of 1988 in a Madurai Hospital in India of 100 cases of consecutive burn victims, found that the majority of these cases were caused by suicide and found to be grouped into three categoriesthose with marital problems (36/100), other stressful family and life circumstances (26/100), and dowry related problems (5/100) (16). This information was gathered by contacting the survivors of suicide attempts, family members of those who committed suicide, in addition to the Department of Plastic Surgery and Forensic medicine reviewing each case individually and deciding whether they were likely accidental, suicidal or homicidal (16). One of the differences found between the accidental versus suicidal burns were that 15 of the cases were mentally ill in the suicidal group while only three in the accidental group. Among the suicide group, eight cases had made prior suicide attempts but not by burn injury (two of which were psychiatrically ill, and other two who were physically ill) (16). This suggests that in India like many other countries, suicide is associated with psychiatric pathology.

\subsubsection{Adolescent suicide in India}

In India, each year greater than 100,000 people commit suicide with a gradual upward trend being noted (17). With the highest percentage of those being below the age of 30, accounting for about 37\% of all suicides in India in $2009(19,21)$ it is important to identify factors that may differentiate this group. In a study of 149 suicide attempters, adolescents differentiated themselves from the other age groups in that $90 \%$ of them made their attempts impulsively with only $9 \%$ of the attempts being of high potential in terms of risk (26). Within this adolescent population, the most common predisposing factor to suicide was reported childhood trauma while mental illness was the least when compared to other age groups (27). In addition, all of these adolescents reported experiencing negative life events (27), which typically included failing examinations and minor violations of discipline with anticipation of negative consequences $(27,28)$. Among the listed reasons, "illness," "family problems," and "failure in examination" were the main reasons for suicide among children (23). In comparison, adults that attempted suicides typically had an underlying psychiatric illness and the methods they used were considered more serious attempts, while suicide attempts by adolescents in India were more impulsive and due to "anomalous life conditions" (1).

\subsubsection{Immigration and suicide}

Although there are no studies found that specifically examined adolescent suicide among Indian adolescents in the US, one study published in the British Journal 
of Psychiatry looked at the suicide patterns among immigrants in Britain that immigrated from the Indian subcontinent. From 1970 to 1978, it was found that 145 Indian men and 86 Indian women committed suicide in England and Wales which accounted for $1.8 \%$ of male death and $2.7 \%$ of female death among Indians in England and Wales (25). When compared to the same period and rates of suicide in England and Wales, only $0.8 \%$ and $0.6 \%$ respectively of male and female deaths were accounted for by suicide (25). Among both genders, suicide mortality was higher among young Indians, specifically Indian women between the ages of 15-24 who had over an $80 \%$ higher suicide rate than women of corresponding ages (25).

When individuals from different cultures immigrate to a host country, the immigrants assume similar suicide rates and methods to members of the host culture (29). Generally, suicide rates among first generation immigrants are lower and most resemble rates typical of their country of origin (30) with subsequent generations have increased rates comparable to North American trends (31). Some factors that contribute to assimilation and may impact these rates include acculturative stress, process of acculturation, risk factors (i.e., single, drug use and rejection by host culture) and protective factors (family support, belonging to an ethnic community, being married, living in a large city) (29). The stress associated with immigration, whether or not there is an underlying mental illness, can lead to severe and possibly chronic behavioral issues including alcohol and illicit drug abuse, depression, anxiety, and posttraumatic stress disorder which subsequently place these individuals at higher risk for suicide (32). First generation immigrant populations within North America tend to choose methods of suicide that are influenced by their cultural heritage (29), however, it was noted that Indian immigrants tend to switch methods of suicide from poisoning with pesticides to hanging, which may reflect the lack of availability of pesticides in the host countries or may indicate that the host culture has an influence on the immigrant (33).

\subsubsection{Preventing suicidal behavior in Indian adolescents}

For many who engage in suicidal behavior, there is often an appropriate alternative resolution to the precipitating problems that lead to suicide (18). Non-governmental organizations (NGOs) are a large support system that should be used to help support suicidal individuals (18). NGOs can serve not only as an entry point for individuals requiring professional services, but also provide education, raise awareness in the public and establish intervention programs (18). Reducing access to lethal means including pesticide and alcohol which are associated with high rates of suicide in India would be part of the national plan for suicide prevention (18). Encouraging and promoting responsible reporting of suicide and issues relating to suicide, supporting NGOs, improving the capacity of primary care workers and mental health specialists, and providing support for those who suffer from the loss of someone who 
committed suicide should all be part of the solution to preventing suicide in India (18). In general, an overall improvement in psychoeducation and subsequent increased awareness of people in the community concerning mental illness would likely decrease the stigma and hopefully improve suicide outcomes.

Features of impulsivity which include poor impulse control, quick provocation, and disregard of external constraints are at higher levels at age 17 and with a slower decline with age in those who attempted suicide when compared to non-attempters (34). Therefore, it is imperative that public health interventions such as restricting access to pesticides (35), increased surveillance, training and community action on the use of pesticides (i.e., safe storage and proper dilutions) (36), and stricter alcohol laws may prevent many deaths caused by suicide in India. Secondary prevention is also very important and includes recognizing and treating psychiatric disorders, particularly mood disorders and alcohol abuse (37) which would also decrease suicide risk.

The well-known theory by Emile Durkheim stresses the importance of community and social networking as being protective against suicide (38). He felt that difference in suicide rates seen between cultures was an inverse function of the level of social integration, such that individuals with strong social bonds had a lower risk for suicide then those with less developed networks. Supporting this theory, living in a large city is protective against suicide. Factors that need to be strengthened in Indian adolescents that immigrate to another county are maintaining culture and establishing an ethnic community (29). It is this ethnic community that appears to similarly serve as the family unit for immigrants.

\section{References}

1. Kar N. Profile of risk factors associated with suicide attempts: A study from Orissa, India. Indian J Psychiatry 2010;52(1):48-56.

2. Anderson RN, Smith BL. Deaths: leading causes from 2001. Natl Vital Stat Rep 2003;52(9):1-85.

3. Eaton DK, Kann L, Kinchen S, Shanklin S, Flint KH, Hawkins J, et al. U.S. Department of Health and Human Services Center for Disease Control and Prevention. Youth Risk Behavior SurveillanceUnited States, 2011. MMWR Surveill Summ 2012;61(4):1-163.

4. Marttunen M, Pelkonen M. Psychiatric risk factors for adolescent suicide: A review. Psychiatr Fenn 2000;31:110-25.

5. Shafii M, Steltz-Lenarsky J, Derrick AM, Beckner C, Whittinghill JR. Comorbidity of mental disorders in the post-mortem diagnosis of completed suicide in children and adolescents. J Affect Disord 1988;15:277-83.

6. Marttunen M, Aro H, Henriksson M, Lönnqvist JK. Mental disorders in adolescent suicide: DSM-III-R axes I and II among 13 to 19 year olds in Finland. Arch Gen Psychiatry 1991;48(9):834-9.

7. Avasthi A. Indianizing psychiatry - Is there a case enough? Indian J Psychiatry 2011;53(2):111-20.

8. Sharaf AY, Thompson EA, Walsh E. Protective effects of self-esteem and family support on suicide risk behaviors among at-risk adolescents. J Child Adolesc Psychiatr Nurs 2009;22(3):160-8. 
9. Cheng ST, Chan AC. Multiple pathways from stress to suicidality and the protective effect of social support in Hong Kong adolescents. Suicide Life Threat Behav 2007;37(2):187-96.

10. Campion J, Bhugra D. Experiences of religious healing in psychiatric patients in south India. Soc Psychiatry Psychiatr Epidemiol 1997;32(4):215-21.

11. Kakar DN. Primary Health Care and Traditional Medicine Practitioner. Sterling, New Delhi 1988.

12. Mohan B. Social Psychiatry in India. Minerva press, Calcutta, 1972.

13. Khan MM. Suicide on the Indian subcontinent. Crisis 2002;23(3):104-7.

14. Khan MM. Suicide and attempted suicide in Pakistan. Crisis 1998;19(4):172-6.

15. Latha KS, Geetha N. Criminalizing suicide attempts: can it be a deterrent? Med Sci Law 2004;44(4):343-7.

16. Rao AV, Mahendran N, Gopalakrishnan G, Reddy TK, Prabhakar ER, Swaminathnan R, et al. One hundred female burn cases: A study in suicidology. Indian J Psychiatry 1989;31(1):43-50.

17. National Crime Records Bureau. Accidental Deaths and Suicide in India 2008. Ministry of Home Affairs, Government of India 2008;168-89. http://ncrb.nic.in/ADSI2008/suicides-08.pdf. Accessed July 23, 2012.

18. Vijaykumar L. Suicide and its prevention: The urgent need in India. Indian J Psychiatry 2007;49(2):81-4.

19. Reddy MS. Suicide incidence and epidemiology. Indian J Psychol Med 2010;32(2):77-82.

20. World Health Organization. Mental health. Suicide rates (per 100,000), by gender, USA, 1955-2005. http://www.who.int/mental_health/media/unitstates.pdf. Accessed July 24, 2012.

21. World Health Organization. Mental health. Suicide rates (per 100,000), by gender, India, 1980-2009. http://www.who.int/mental_health/media/indi.pdf. Accessed July 24, 2012.

22. Mayer P, Ziaian T. Suicide, gender and age variations in India. Are women in Indian society protected from suicide? Crisis 2002;23(3):98-103.

23. Government of India. Accidental Deaths and Suicide in India. National Crime Records Bureau. Ministry of Home Affairs: 2010.

24. Vijayakumar L, Nagaraj, Pirkis J, Whiteford H. Suicide in developing countries (1): Frequency, distribution and association with socioeconomic indicators. Crisis 2005;26(3):104-11.

25. Soni Raleigh V, Bulusu L, Balarajan R. Suicides among immigrants from the Indian subcontinent. Br J Psychiatry 1990;156:46-50.

26. Shaffer D. Suicide in childhood and early adolescence. J Child Psychol Psychiatry 1974;15(4): 275-91.

27. Kar N, Pany M, Mishra BN, Sengupta J, Das I. Risk factors of adolescent suicide attempt. J East Zonal Branch Indian Psychiatr Soc 1996;1:17-22.

28. Mukherji A. Around 6,000 students committed suicide in 2006. The Times of India. http:// timesofindia.indiatimes.com/Around_6000_students_committed_suicide_in_2006/ articleshow/2872298.cms. Accessed November 25, 2011.

29. Greenfield B, Daniel L, Harnden B. Immigration and suicide: An overview. In: Sher L, Vilens A, eds. Immigration and Mental Health: Stress, Psychiatric Disorders and Suicidal Behavior among Immigrants and Refugees. New York: Nova Science, 2010:9-20.

30. Sainsbury P, Barraclough B. Differences between suicide rates. Nature 1968;220:1252.

31. Pena JB, Wyman PA, Brown CH, Matthieu MM, Olivares TE, Hartel D, et al. Immigration generation status and its association with suicide attempts, substance use, and depressive symptoms among latino adolescents in the USA. Prev Sci 2008;9(4):299-310.

32. Sher L. A model of suicidal behavior among immigrants with psychiatric disorders. In: Sher L, Vilens A, eds. Immigration and Mental Health: Stress, Psychiatric Disorders and Suicidal Behavior among Immigrants and Refugees. New York: Nova Science Publishers, 2010, pp. 1-8.

33. Lester D. Suicide in emigrants from the Indian subcontinent. Transcult Psychiatry 2000;37(2): 243-54. 
34. Kasen S, Cohen P, Chen H. Developmental course of impulsivity and capability from age 10 to age 25 as related to trajectory of suicide attempt in a community cohort. Suicide Life Threat Behav 2011;41(2):180-92.

35. Patel V, Ramasundarahettige C, Vijayakumar L, Thakur JS, Gajalakshmi V, Gururaj G, et al. Million death study collaborators. Suicide mortality in India: A nationally representative survey. Lancet 2012;379(9834):2343-51.

36. World Health Organization. Suicide huge but preventable public health problem, says WHO. Indian J Med Sci 2004;58(9):409-11.

37. Pelkonen M, Marttunen M. Child and adolescent suicide epidemiology, risk factors, and approaches to prevention. Paediatr Drugs 2003;5(4):243-65.

38. Durkheim E. Suicide: A Study in Sociology. London: Routeldge, 1952. 
